\title{
Facteurs déterminant la variabilité spatiale de la biomasse herbacée dans la zone soudano-guinéenne du Bénin
}

\author{
Ismaïla TOKO* et Brice SINSIN \\ Laboratoire d'Ecologie Appliquée, Université d'Abomey-Calavi, 03BP1974 Cotonou, Bénin. \\ *Auteur correspondant,Email : ismael_toko@yahoo.fr; Tel : 0022997772872
}

\section{RESUME}

L'influence de la topographie, des conditions édaphiques, des types biologiques, du biovolume des herbacées, de la densité et du recouvrement des ligneux sur la variation spatiale de la biomasse herbacée à l'échelle de petits bassins versants a été étudiée dans la zone soudano-guinéenne au centre du Bénin. La biomasse herbacée a été coupée suivant la méthode de coupe rase dans 40 placeaux de $10 \mathrm{~m}$ x $10 \mathrm{~m}$. Au total, 40 relevés phytosociologiques ont été effectués suivant la méthode sigmatiste de Braun-Blanquet dans des placeaux de $30 \mathrm{~m}$ x $30 \mathrm{~m}$. Les modèles de régressions linéaires et de régression multiple ont été testés avec le logiciel Statview. Les plus faibles biomasses sont obtenues sur les interfluves alors que les dépressions sont les plus productives. Les sols argileux et limoneux sont plus productifs que les sols sableux. La biomasse herbacée varie significativement selon les types de formations végétales et les types biologiques. Les plus fortes biomasses herbacées sont obtenues dans les savanes herbeuses et arborées. Les hémicryptophytes et les thérophytes fournissent plus de $90 \%$ de la biomasse herbacée. La biomasse des hémicryptophytes est respectivement 2 fois, 8 fois et 10 fois supérieure à celle des thérophytes, des chaméphytes et des géophytes.

(C) 2011 International Formulae Group. All rights reserved.

Mots clés : Biomasse herbacée, biovolume, topographie, sols, bassin supérieur du fleuve Ouémé, Bénin.

\section{INTRODUCTION}

La végétation herbacée des savanes tropicales fournit entre 75 et $90 \%$ de la biomasse totale des écosystèmes (Garnier et Dajoz, 2001). Les facteurs biotiques notamment la composition floristique, la densité et la surface terrière des ligneux, le recouvrement de la canopée et les facteurs abiotiques tels que le climat, la topographie et les conditions édaphiques influent sur la variation de la biomasse herbacée (Hansen et al., 2000; Van der Welle, 2003; Squires et Bennett, 2004; Toko et Sinsin, 2008). Certains auteurs (Hutchinson, 1996 ; Keya,
1998) estiment que les facteurs biotiques sont plus déterminants que les facteurs abiotiques dans la variation de la biomasse herbacée. Par contre, pour d'autres auteurs, les processus naturels tels que le climat, la pluviosité et la nature du substrat édaphique jouent un rôle déterminant dans le fonctionnement et la dynamique des systèmes écologiques (Lepart, 1997 ; Marage, 2004). Ainsi, la variation de la biomasse suivant la topographie est surtout liée aux caractéristiques physico-chimiques et à la disponibilité en eau des sols (Sinsin et al., 1989 ; Agonyissa et Sinsin, 1998 ; Houinato et al., 2003 ; Cardinale et al., 2004 ; Orthmann, 
2005 ; Toko et Sinsin, 2008). Toutefois, la disponibilité en eau des sols dépend non seulement de la quantité de pluie mais surtout de sa bonne répartition; ce qui influence de facto la variation de la biomasse herbacée (Ellis et Swift, 1988 ; Lejoly et Sinsin, 1993 ; Houinato et al., 2003 ; Sawadogo et al., 2005). Par ailleurs, les plus fortes biomasses sont obtenues dans les formations végétales à faible densité de ligneux. Ainsi, les savanes herbeuses et arbustives sont plus productives que les autres types de formations végétales (Lejoly et Sinsin, 1994 ; Agonyissa et Sinsin, 1998 ; Sinsin et al., 2000 ; Houinato et al., 2003).

Au cours de ces dernières décennies, de nombreuses études concernant la productivité des pâturages ont été réalisées dans la région soudanienne du Bénin. Ainsi, plusieurs travaux (Lejoly et Sinsin, 1993 ; Houinato et al., 2003) ont abordé l'influence de la pluviosité sur la productivité des pâturages. D’autres par contre (Lejoly et Sinsin, 1994 ; Agonyissa et Sinsin, 1998; Sinsin et al., 2000 ; Houinato et al., 2003) ont mis en évidence l'effet dépressif du couvert ligneux sur la végétation herbacée. Par ailleurs, certaines études (Sinsin et al., 1989; Agonyissa et Sinsin, 1998 ; Houinato et al., 2003 ; Orthmann, 2005) se sont focalisées sur le rôle des facteurs édaphiques et topographiques dans la variation de la biomasse herbacée. Enfin, d'autres auteurs (Sinsin et Saïdou, 1998; Houinato et al., 2003) ont travaillé sur l'impact des feux de végétation sur la productivité des pâturages.

Tous ces travaux ont abordé séparément les facteurs déterminant la productivité des pâturages alors que l'ensemble des processus mis en jeu dans ce fonctionnement devrait être pris en compte de façon intégrée à l'échelle des bassins versants. Cette étude a pour but de déterminer le facteur le plus prépondérant parmi la topographie, les conditions édaphiques (types, profondeur et temps d'infiltration des sols), les types biologiques, le biovolume des herbacées, la densité et le recouvrement des ligneux, dans la variation spatiale de la biomasse herbacée à l'échelle de petits bassins versants.

\section{MATERIEL ET METHODES \\ Cadre de l'étude}

Les données ont été collectées dans le bassin supérieur du fleuve Ouémé au centre du Bénin entre $8^{\circ}$ et $9^{\circ} 50$ de latitude nord et entre $2^{\circ}$ et $2^{\circ} 50$ de longitude Est (Figure 1). Il est sous 1'influence du climat soudanoguinéen. La pluviosité et la température moyenne annuelle sont respectivement de $1150 \mathrm{~mm}$ et de $27{ }^{\circ} \mathrm{C}$. Le substratum géologique dominant dans la région est constitué de formations cristallines très anciennes de type 'Dahomeyen". Les sols sont en majorité des sols ferrugineux tropicaux et accessoirement des sols ferralitiques dans la partie Nord-Ouest (Faure, 1977). Le paysage est essentiellement constitué de pénéplaine cristalline d'altitude moyenne de $300 \mathrm{~m}$. Le relief est dominé par une série d'inselbergs orientés nord-sud dont le plus élevé est le mont Soubakpérou (620 $\mathrm{m})$. Le réseau hydrographique est constitué par plusieurs rivières dont les plus importantes sont l'Ouémé et la Térou. Le secteur d'étude est situé dans le Centre Régional d'Endémisme Soudanien (White, 1983) et est considéré comme une zone de transition Guinéo-Soudanienne (Adjanohoun et al., 1989) et appartient aux districts phytogéographiques de Bassila et du BorgouSud (Adomou et al., 2006). La végétation est composée d'îlots de forêts denses sèches, de galeries forestières, de forêts claires, de savanes boisées, de savanes arborées et arbustives et de savanes saxicoles. La classification réalisée sur la base des images satellites LANDSAT de 2000, montre que la végétation du secteur d'étude est composée de $25 \%$ de formations forestières et de $69 \%$ de formations savanicoles (IMPETUS, 2005).

\section{Choix des petits bassins versants et des placeaux}

Les principaux critères de choix des petits bassins versants sont l'importance et la 
densité du réseau hydrographique, la longueur des versants, l'accessibilité des sites, la présence d'aire protégée et le degré d'anthropisation.

A l'intérieur des petits bassins versants, plusieurs transects transversaux allant du bas de versant vers l'interfluve, sont choisis dans le but de recouper la diversité des conditions de sol et de topographie. 3 à 5 placeaux sont installés suivant les variations de la toposéquence et des faciès de végétation. Au total, 3 à 4 transects de 1 à $6 \mathrm{~km}$ de long et 6 à 12 placeaux ont été installés par petit bassin versant. Pour ressortir l'influence des conditions de drainage du sol, quelques placeaux sont installés suivant le regard de drainage dans les drains.

\section{Quantification de la biomasse herbacée}

La méthode classique de coupe rase a été utilisée pour la quantification de la biomasse herbacée (Houinato et al., 2001; Toko et Sinsin, 2008).

La biomasse est coupée à l'intérieur des placeaux de $10 \mathrm{~m}$ x $10 \mathrm{~m}$ installés dans les dépressions, au bas des versants, sur les miversants et les interfluves des toposéquences suivant la méthode de transect. Au total, 40 placeaux de $10 \mathrm{~m}$ x $10 \mathrm{~m}$ ont été installés dans le secteur d'étude.

La biomasse est récoltée mensuellement de mai à novembre. Les espèces sont coupées au ras du sol à l'aide d'un sécateur de jardinier dans 7 placettes de $1 \mathrm{~m}^{2}$ choisies au hasard dans chaque placeau. La biomasse des trois premières périodes (juin, juillet et août) de coupe est triée afin de séparer les poaceae des autres espèces. Par contre, pour les trois dernières périodes de coupe (septembre, octobre et novembre), la biomasse de chaque espèce est mesurée afin de déterminer la contribution spécifique de chaque espèce ou de chaque type biologique. Le poids frais des différentes catégories (poaceae, cyperaceae et forbes) est mesuré à l'aide de pesons. Un échantillon de $100 \mathrm{~g}$ de chacune des catégories est mis dans des sachets et pré séché sur le terrain. Ces échantillons sont ensuite emballés dans des enveloppes de productivité puis mis à l'étuve à $60{ }^{\circ} \mathrm{C}$ pendant 72 heures jusqu'à l'obtention du poids sec constant.

\section{Collecte des données floristiques}

Les relevés phytosociologiques sont effectués suivant la méthode sigmatiste de Braun-Blanquet (Weber et al., 2000) dans 40 placeaux de $30 \mathrm{~m}$ x $30 \mathrm{~m}$. L'aire de relevé est de $100 \mathrm{~m}^{2}(10 \mathrm{~m} \times 10 \mathrm{~m})$ pour la strate herbacée et de $900 \mathrm{~m}^{2}$ (30 m x $30 \mathrm{~m}$ ) pour les strates arbustive et arborée.

L'identification des types biologiques des espèces a été réalisée sur la base des travaux de Raunkiaer (Djego et Sinsin, 2006).

La hauteur moyenne des espèces de la strate herbacée a été mesurée à l'aide d'un décamètre.

Les données dendrométriques ont concerné le nombre d'individus de chaque espèce, le diamètre des ligneux de $\mathrm{dbh} \geq 10$ $\mathrm{cm}$ et le degré d'ouverture de la strate arborescente. Le degré d'ouverture de la strate arborescente dans les placeaux a été apprécié par cotation visuelle de ciel ouvert à travers le feuillage. Les classes proposées par Godron et al. (1983 in Kokou et al., 2000) ont été adoptées :

$1:$ Strate fermée (recouvrement $>90 \%$ )

2 : Strate peu ouverte (recouvrement entre 75 et $90 \%$ )

3: Strate assez ouverte (recouvrement entre 50 et $75 \%$ )

4: Strate ouverte (recouvrement entre 25 et $50 \%$ )

5 : Strate très ouverte (recouvrement entre 10 et $25 \%$ )

6 : Strate extrêmement ouverte (recouvrement entre 0 et $10 \%$ )

7: Strate totalement ouverte (recouvrement égal à $0 \%$ ).

\section{Collecte des données topographiques et pédologiques \\ Les différentes positions}

topographiques (dépression, bas de versant, mi-versant et interfluve) sont directement 
notées sur le terrain. La mesure de la pente est faite à l'aide du clinomètre et d'une tige sur laquelle on attache une banderole fluorescente à la hauteur de l'œil du viseur. Une fois la tige positionnée au centre du placeau, le viseur lit directement la pente $(\%)$ sur le clinomètre en visant la banderole.

La texture (sable, limon et argile) des sols est déterminée par la méthode tactile grâce au concours des pédologues du projet IMPETUS, sur des échantillons de sol prélevés dans les 10 premiers $\mathrm{cm}$ du sol, dans les 4 coins et au centre de chaque placeau.

La profondeur des sols est mesurée dans des fosses pédologiques de $50 \mathrm{~cm}$ de côté et de profondeur comprise entre 100 et $150 \mathrm{~cm}$ car la roche mère des sols de la région est généralement au delà de $100 \mathrm{~cm}$. Dans ces fosses, la vitesse de retrait des eaux est mesurée après chaque précipitation. Pour ce faire, la codification du Tableau 1 a été adoptée.

\section{Traitement des données}

La biomasse herbacée $(\mathrm{Bh})$ est calculée suivant la formule : $\mathrm{Bh}(\mathrm{t} \mathrm{MS} / \mathrm{ha})=(1 / 7) \mathrm{x}$ 10000/S x $\Sigma$ PFi (g) x PS (g) /100 g. 7: nombre de placettes par placeau; $\mathrm{PFi}=$ poids frais en gramme mesuré sur le terrain pour la placette $\mathrm{i}$; PS= poids sec en gramme obtenu après séchage à l'étuve pour un échantillon de $100 \mathrm{~g} \mathrm{du}$ placeau; 10000/S : superficie inventoriée rapportée à 1'hectare.

Le biovolume $\mathrm{Bv}\left(\mathrm{m}^{3} / \mathrm{ha}\right)$ est calculé selon la formule : $\mathrm{Bv}=\Sigma \mathrm{RM}_{\mathrm{i}} \times \mathrm{H}_{\mathrm{i}} \times 10000 / \mathrm{S}$ où $\mathrm{RM}_{\mathrm{i}}=$ Recouvrement moyen de l'espèce $\mathrm{i}$, obtenu d'après l'échelle d'abondancedominance de Braun-Blanquet (Weber et al., 2000), $H_{i}=$ Hauteur de l'espèce $\mathrm{i}(\mathrm{m})$ et $10000 / \mathrm{S}$ : superficie inventoriée rapportée à l'hectare.

La richesse spécifique: C'est le nombre d'espèces présentes dans une aire déterminée $\left(900 \mathrm{~m}^{2}\right.$ pour la présente étude) de la phytocénose.

La densité (D) des ligneux a pour formule : $\mathrm{D}=\mathrm{N} \times 10000 / \mathrm{S}$ où $\mathrm{D}:$ nombre de tiges/ha ; $\mathrm{N}:$ nombre de tiges de $\mathrm{dbh} \geq 10 \mathrm{~cm}$ et 10000/S : superficie inventoriée rapportée à l'hectare.

La surface terrière moyenne est calculée selon la formule : $\mathrm{G}_{\mathrm{i}}=\sum 1 / 4 \Pi \mathrm{D}^{2} \mathrm{x}$ $10000 / \mathrm{S}$ où $\mathrm{G}_{\mathrm{i}}$ est en $\mathrm{m}^{2} / \mathrm{ha} ; \mathrm{D}$ : diamètre (m) ; 10000/S : superficie inventoriée rapportée à 1 hectare.

Le test de corrélation de Spearman et les tests d'homogénéité de Kruskal-Wallis et de Mann-Whitney ont servi à mesurer l'effet des variables environnementales stationnelles (situation topographique, types de sols, profondeur et humidité du sol, types de végétation, densité, surface terrière et taux de recouvrement des ligneux, biovolume de la strate herbacée) sur la biomasse herbacée. Le test de Kruskal-Wallis est un test nonparamétrique sur $\mathrm{k}$ échantillons indépendants. Il a permis de faire l'étude des liaisons entre la biomasse et les différents facteurs à k classes. Le test de Mann-Whitney est aussi un test non paramétrique d'identité portant sur deux échantillons indépendants issus de variables numériques ou ordinales.

Les modèles de régressions linéaires ont été utilisés pour prédire l'évolution de la biomasse herbacée en fonction des variables environnementales stationnelles.

Le test de régression multiple a été utilisé pour déterminer le facteur le plus prépondérant dans la variation de la biomasse herbacée. Le logiciel Statview a été utilisé pour les différents tests.

\section{RESULTATS}

Variation de la biomasse herbacée suivant la topographie et les types de sols

La variation de la biomasse herbacée suivant la topographie montre que les dépressions sont plus productives que les interfluves et les versants (Figure 2a). Cette productivité varie en moyenne de 3,5 t MS/ha dans les dépressions à 2,6 t MS/ha sur les interfluves et 2,3 t MS/ha sur les versants. Le test de Kruskal-Wallis montre une différence significative $(\mathrm{P}=0,0010)$ entre les dépressions, les interfluves et les versants. Le test de Mann-Whitney montre une différence 
significative entre les interfluves et les dépressions $(\mathrm{P}=0,0087)$ d'une part et entre les versants et les dépressions $(P=0,0002)$ d'autre part. Par contre, aucune différence significative n'est notée entre les interfluves et les versants. Par ailleurs, cette variation suivant la topographie diffère parfois selon les toposéquences. Ainsi, il existe des toposéquences sur lesquelles les faibles biomasses sont obtenues sur les interfluves alors que sur d'autres, ce sont les dépressions qui fournissent les plus faibles biomasses.

La biomasse obtenue sur les différents types de sol montre que les sols limoneux et les sols argileux sont les plus productifs. Par contre, la biomasse est très faible sur les sols sableux (Figure 2b). Le test de Mann-Whitney montre une différence significative $(\mathrm{P}<$ 0,0001) entre les sols argileux et sableux d'une part et entre les sols limoneux et sableux d'autre part. Par contre, aucune différence n'est notée entre les sols argileux et limoneux.

\section{Variation de la biomasse herbacée suivant les formations végétales et les types biologiques \\ Les plus fortes biomasses herbacées} sont obtenues dans les savanes herbeuses et arborées. Elles sont suivies par les forêts galeries dégradées. Par contre, la biomasse herbacée des savanes boisées et des forêts claires est très faible (Figure $3 \mathrm{a}$ ). La biomasse produite dans les forêts claires équivaut au quart de la biomasse des savanes herbeuses, au tiers de celle des savanes arborées et des forêts galeries dégradées et enfin à la moitié de celle des savanes boisées. Le test de Kruskal-Wallis montre une différence hautement significative $(\mathrm{P}<0,0001)$ entre les biomasses des formations végétales.

Concernant la biomasse des types biologiques des herbacées, les hémicryptophytes (2,0 t MS/ha) produisent respectivement 2 fois, 8 fois et 10 fois plus de biomasse herbacée que les thérophytes $(1,1 \mathrm{t}$ MS/ha), les chaméphytes (0,4 t MS/ha) et les géophytes (0,2 t MS/ha) (Figure 3b). Les hémicryptophytes et les thérophytes produisent plus de $90 \%$ de la biomasse totale du secteur d'étude.

Modèles de régression linéaire entre les facteurs biotiques, abiotiques et la variation spatiale de la biomasse herbacée

Plusieurs facteurs biotiques et abiotiques interagissent dans la variabilité spatiale de la biomasse herbacée. Le test de corrélation de Spearman a montré que l'évolution de la biomasse herbacée est fortement liée à certaines variables environnementales notamment la profondeur du sol ( $\mathrm{p}<0,0001)$, le temps d'infiltration ( $\mathrm{p}$ $=0,0011)$, le biovolume de la strate herbacée $(\mathrm{p}<0,0001)$, le recouvrement des ligneux $(\mathrm{p}<$ $0,0001)$ et la densité des ligneux de $\mathrm{dbh} \geq 10$ $\mathrm{cm}(\mathrm{p}=0,0177)$. Par contre, aucune relation claire n'est établie $(\mathrm{p}>0,05)$ entre la variation de la biomasse herbacée et la topographie, la richesse spécifique et la surface terrière des ligneux. Le Tableau 2 présente les résultats de l'analyse de variance (ANOVA) entre la biomasse herbacée et les variables environnementales testées.

Les modèles de régression linéaire obtenus à partir de l'évolution de la biomasse herbacée et les facteurs biotiques et abiotiques ont pour formules :

$\mathrm{Y}_{\text {biomasse }}=-0,046 \mathrm{X}_{\text {recouvrement des ligneux }}+5,012$; $\mathrm{R}^{2}=0,638 ; \mathrm{p}<0,0001$

$\mathrm{Y}_{\text {biomasse }}=1,397^{\mathrm{E}-4} \mathrm{X}_{\text {biovolume }}+1,75$; $\mathrm{R}^{2}=0,525 ; \mathrm{p}<0,0001$

$\mathrm{Y}_{\text {biomasse }}=0,059 \mathrm{X}_{\text {profondeur du sol }}-0,613$; $\mathrm{R}^{2}=0,418 ; \mathrm{p}<0,0001$

$\mathrm{Y}_{\text {biomasse }}=0,009 \mathrm{X}_{\text {temps d'infiltration }}+2,553$; $\mathrm{R}^{2}=0,172 ; \mathrm{p}=0,0051$

$\mathrm{Y}_{\text {biomasse }}=-0,007 \mathrm{X}_{\text {densité des ligneux }}+4,882$; $\mathrm{R}^{2}=0,161 ; \mathrm{p}=0,0064$

Les coefficients de variation indiquent respectivement qu'une augmentation d'un centimètre de la profondeur du sol et d'un mètre cube par hectare du biovolume de la strate herbacée entraîne respectivement une augmentation de l'ordre de 0,059 t MS/ha et de $1,397{ }^{\mathrm{E}-4} \mathrm{t}$ MS/ha de la biomasse herbacée. Par ailleurs, une augmentation d'une minute 
du temps d'infiltration des eaux de précipitations entraîne une augmentation de la biomasse herbacée de l'ordre de 0,009 t MS/ha. Par contre, une augmentation respective d'un pourcent du recouvrement des ligneux et d'une tige de ligneux $(\mathrm{dbh} \geq 10 \mathrm{~cm})$ par hectare entraîne respectivement une diminution de 0,046 t MS/ha et de 0,007 t MS/ha de la biomasse herbacée.

La Figure 4 permet d'illustrer l'adéquation entre les observations et l'estimation des valeurs calculées par les modèles.

Les valeurs des coefficients de détermination $\mathrm{R}^{2}$ des différents modèles indiquent que la variation de la biomasse herbacée ne dépend pas uniquement d'une seule variable. Toutefois, la régression multiple révèle que l'ensemble des variables environnementales testées explique $95 \%$ de la variation de la biomasse herbacée $\left(\mathrm{R}^{2}=\right.$ 0,952). La profondeur du sol et le recouvrement des ligneux sont les deux variables environnementales qui influent le plus sur la variation de la biomasse herbacée (Tableau 3). Le temps d'infiltration des eaux de précipitations et le biovolume de la strate herbacée viennent respectivement en troisième et quatrième position. Paradoxalement, aucune corrélation n'est observée entre la densité des ligneux ( $\mathrm{dbh} \geq$ $10 \mathrm{~cm}$ ) et la biomasse herbacée puisque la probabilité p est égale à 0,0737. Ainsi, la variation de la biomasse herbacée dépend en grande partie des quatre variables que sont : la profondeur du sol, le recouvrement des ligneux, le temps d'infiltration des eaux de précipitations et le biovolume de la strate herbacée. Le modèle de régression multiple issu de cette analyse a donc pour formule :

$$
\mathrm{Y}_{\text {biomasse }}=0,042 \mathrm{X}_{\mathrm{PS}}-0,025 \mathrm{X}_{\mathrm{RL}}+
$$
$0,005 \mathrm{X}_{\mathrm{TI}}+4,740^{\mathrm{E}-5} \mathrm{X}_{\mathrm{BH}}$;

$\mathrm{R}^{2}=0,952 ; \mathrm{p}<0,0001$ PS : Profondeur du sol; RL: Recouvrement des ligneux; TI : Temps d'infiltration; $\mathrm{BH}$ : Biovolume des herbacées.

Tableau 1 : Codification de la vitesse de retrait des eaux.

\begin{tabular}{lll}
\hline Code & Intensité & Temps de retrait $(\mathbf{m n})$ \\
\hline 1 & Très faible & $>90$ \\
2 & Assez faible & $30-90$ \\
3 & Faible & $15-30$ \\
4 & Bonne & $5-15$ \\
5 & Très bonne & $1-5$ \\
\hline
\end{tabular}

Tableau 2 : Analyse de variance entre la biomasse et les variables environnementales testées.

\begin{tabular}{lccccc}
\hline $\begin{array}{l}\text { Variables } \\
\text { environnementales }\end{array}$ & $\begin{array}{c}\text { DDL } \\
\text { Profondeur du sol }\end{array}$ & $\begin{array}{c}\text { Somme des } \\
\text { carrés }\end{array}$ & Carré moyen & Valeur de F & Valeur de p \\
Biovolume des herbacées & 1 & 54,625 & 54,625 & 28,742 & $<0,0001$ \\
Recouvrement des ligneux & 1 & 86,116 & 86,116 & 53,021 & $<0,0001$ \\
Densité des ligneux & 1 & 321,580 & 121,580 & 91,258 & $<0,0001$ \\
Temps d'infiltration & 1 & 26,955 & 30,037 & 8,645 & 0,0052 \\
\hline
\end{tabular}


Tableau 3 : Synthèse des coefficients de régression, des valeurs de $t$ et de $p$ obtenu à partir de la régression multiple entre la biomasse et les variables environnementales.

\begin{tabular}{lllllc}
\hline $\begin{array}{l}\text { Variables } \\
\text { environnementales }\end{array}$ & Coefficient & $\begin{array}{l}\text { Erreur } \\
\text { standard }\end{array}$ & $\begin{array}{l}\text { Coefficient } \\
\text { standard }\end{array}$ & Valeur de t & Valeur de p \\
\hline Profondeur du sol & 0,042 & 0,006 & 0,420 & 7,473 & $<0,0001$ \\
Recouvrement des ligneux & $-0,025$ & 0,005 & $-0,444$ & $-4,978$ & $<0,0001$ \\
Temps d'infiltration & 0,005 & 0,002 & 0,242 & 3,328 & 0,0019 \\
Biovolume des herbacées & $4,740^{\mathrm{E}-5}$ & $1,471^{\mathrm{E}-5}$ & 0,279 & 3,222 & 0,0026 \\
Densité des ligneux & 0,002 & 0,001 & 0,135 & 1,838 & 0,0737 \\
\hline
\end{tabular}

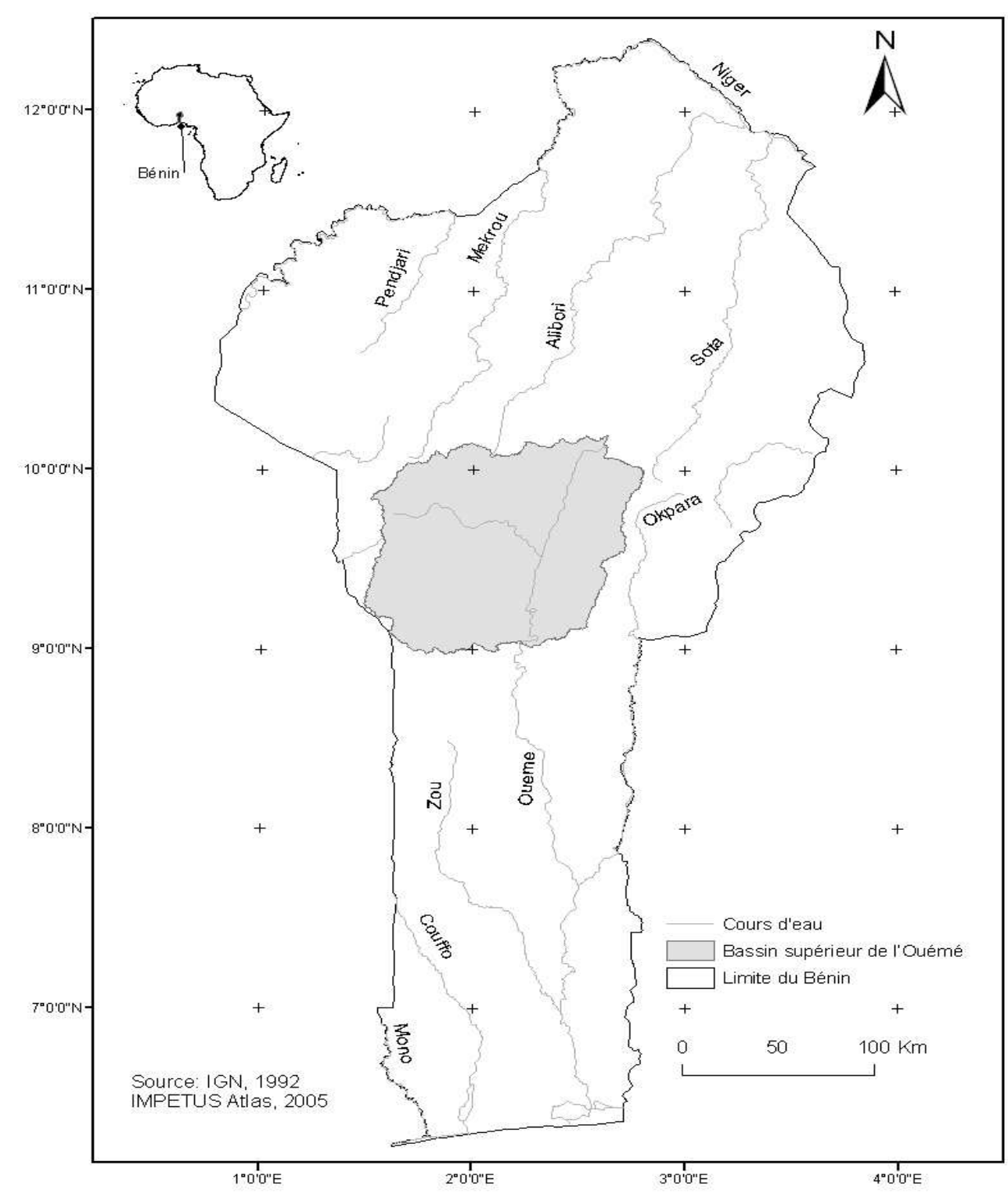

Figure 1 : Carte de localisation du bassin supérieur du fleuve Ouémé. 


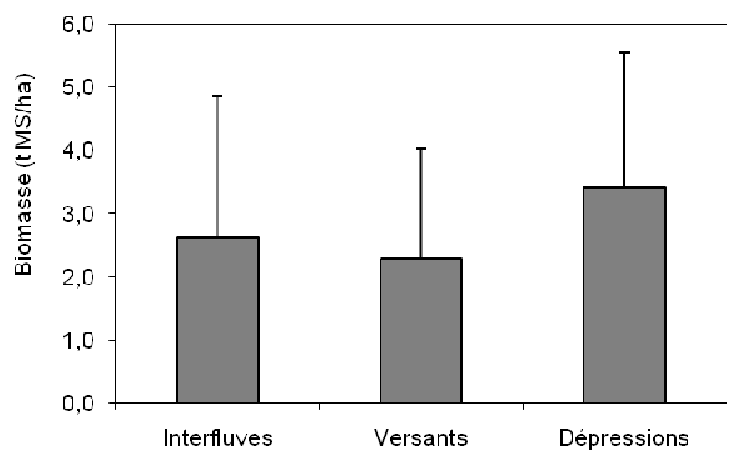

(a) Situation topographique

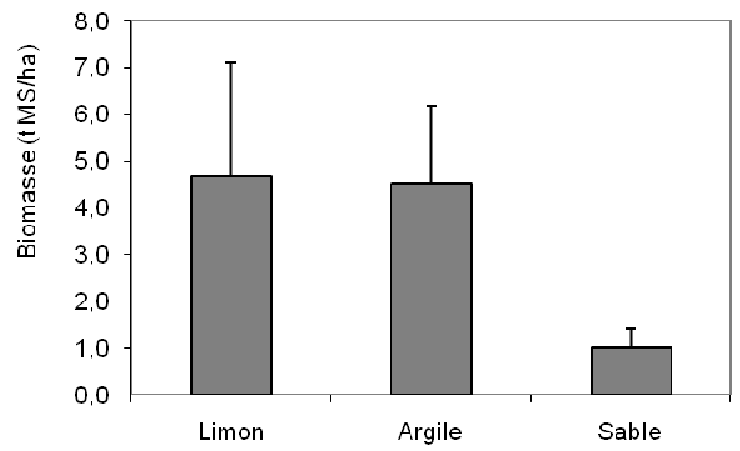

(b) Types de sols

Figure 2 : Variation de la biomasse herbacée suivant les facteurs abiotiques.

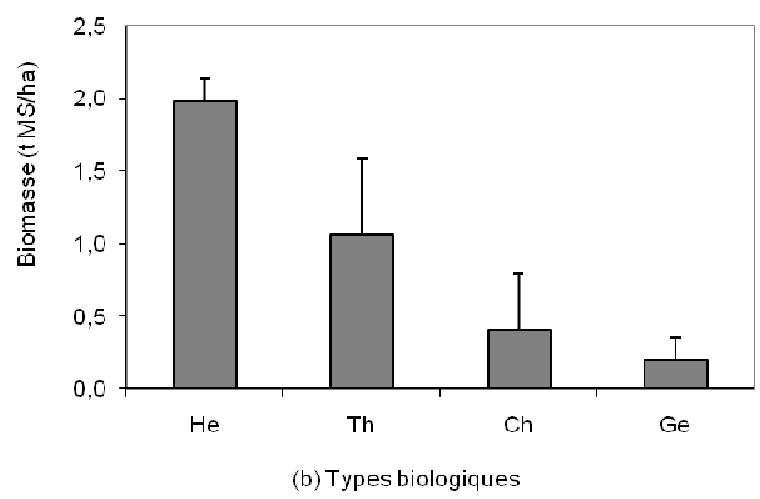




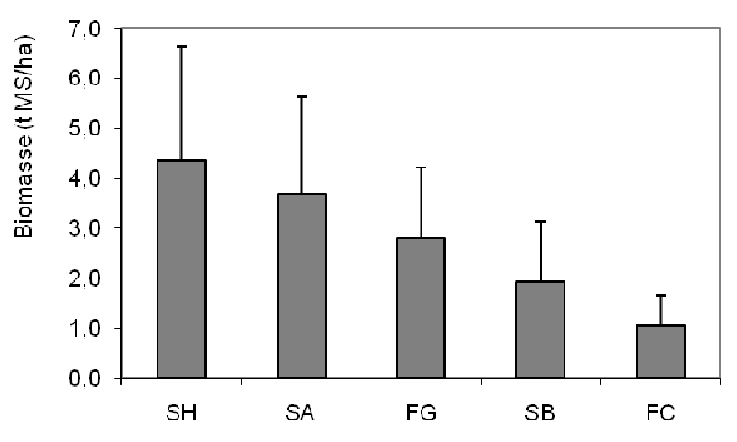

(a) Formations végétales

Figure 3 : Variation de la biomasse herbacée suivant les facteurs biotiques.

SH : Savanes herbeuses ; SA : Savanes arborées ; SB : Savanes boisées ; FC : Forêts claires ;

FG : Forêts galeries dégradées He: Hémicryptophytes ; Th : Thérophytes ; Ch : Chaméphytes ; Ge : Géophytes.
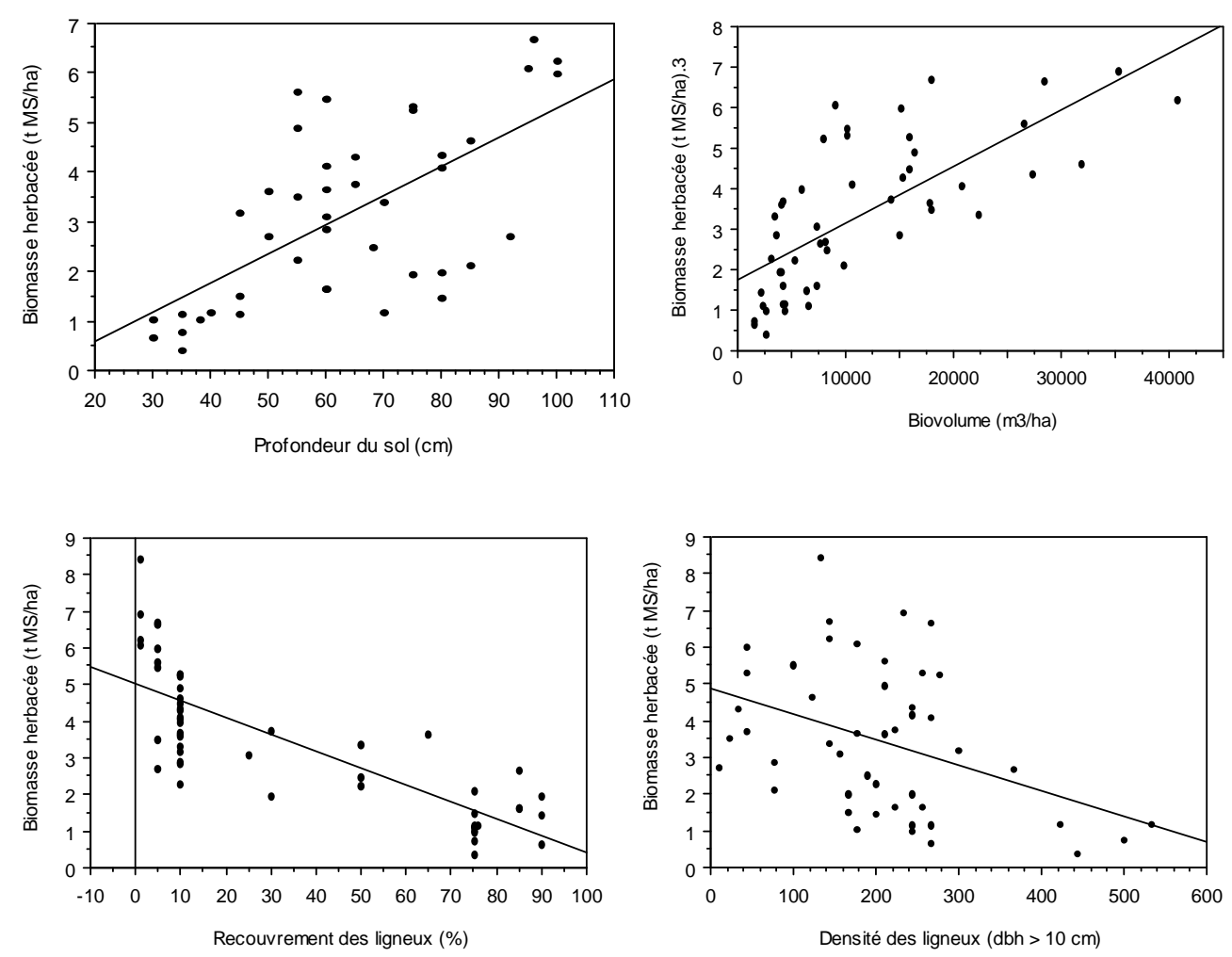


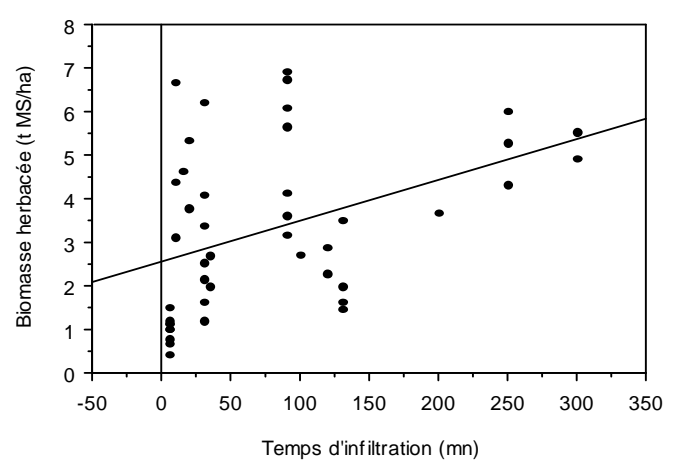

Figure 4 : Droites de régression linéaire entre la biomasse herbacée et les variables environnementales.

\section{DISCUSSION}

Facteurs abiotiques déterminant la variabilité spatiale de la biomasse herbacée

Les conditions édaphiques sont les facteurs abiotiques qui influent le plus sur la variation spatiale de la biomasse herbacée. Les sols sableux produisent moins de biomasse herbacée que les sols argileux et limoneux. La productivité des sols limoneux et des sols argileux est en partie liée à leur profondeur et à leur capacité de rétention d'eau. De ce fait, ces deux types de sols sont favorables à l'installation des graminées pérennes à chaume coriace qui contribuent pour une grande part à la biomasse herbacée. Ces résultats corroborent ceux de plusieurs auteurs (Sinsin et al., 1989; Agonyissa et Sinsin, 1998 ; Houinato et al., 2003 ; Toko et Sinsin, 2008) qui ont rapporté que les caractéristiques physico-chimiques et la disponibilité en eau des sols induisent une forte productivité des savanes. Ainsi, la biomasse herbacée est d'autant plus élevée que le sol est riche en fractions fines. Dans les conditions normales caractérisées par une abondance et une bonne répartition des précipitations, les sols argileux produisent plus de biomasse que les sols sableux (Seghieri et al., 1995 ; Sawadogo et al., 2005). La disponibilité en eau du sol joue alors un rôle important dans la production de la biomasse herbacée car sa présence quasi permanente dans les dépressions, fait que la biomasse produite est élevée et de variance moindre à cause du faible indice d'hétérogénéité de la végétation. La faiblesse de cet indice d'hétérogénéité de la végétation est due à l'élimination des espèces hydrophobes par asphyxie, limitant ainsi la compétition pour les nutriments.

Du point de vue de la topographie, il est difficile de lier la variation de la biomasse herbacée aux toposéquences car des tendances inverses sont parfois observées. La différence de biomasse selon la situation topographique est surtout attribuable à la profondeur et à la disponibilité en eau du sol de même qu'aux types de formations végétales et à leur composition floristique. En effet, sur certaines toposéquences, la productivité est plus élevée sur les interfluves établis sur des sols limoneux très profonds $(100 \mathrm{~cm})$ et occupés par des formations à faible recouvrement de la canopée que dans les dépressions établies sur des sols argileux peu profonds $(35 \mathrm{~cm})$ où la dalle rocheuse est subaffleurante. Par contre, la productivité est faible sur les interfluves occupés par des forêts claires sur des sols sablo-graveleux minces à faible capacité de rétention d'eau. On retient alors que les dépressions et les bas de versant sur sols profonds et humides de texture argileuse ou argilo-limoneuse sont plus productifs que les interfluves sur des sols peu profonds.

Nombre d'auteurs (Sinsin et al., 2000 ; Hansen et al., 2000; Osem et al., 2004 ; 
Orthmann, 2005; Gould et al., 2006) ont montré l'influence de la topographie sur la variation spatiale de la biomasse herbacée. Pour ces auteurs, la biomasse herbacée est inversement proportionnelle à la pente. Ainsi, les dépressions sont plus productives que les interfluves.

Facteurs biotiques déterminant la variabilité spatiale de la biomasse herbacée

La composition floristique, les types de formations végétales, le recouvrement de la canopée et le biovolume de la strate herbacée sont les principaux facteurs biotiques qui déterminent la variation spatiale de la biomasse herbacée dans le secteur d'étude.

La biomasse herbacée évolue suivant un gradient de recouvrement de la canopée. Il existe une corrélation négative entre la quantité de biomasse produite et le recouvrement de la strate arborescente. Ainsi, la biomasse herbacée croît des formations végétales fermées vers les formations végétales ouvertes (Figure 3a). Les strates herbacées des savanes herbeuses et arborées sont donc plus productives que celles des savanes boisées et forêts claires. Paradoxalement, la biomasse produite dans les forêts galeries dégradées est plus importante que celle des savanes boisées et forêts claires. Ces résultats mettent en évidence l'effet dépressif du couvert ligneux et l'importance de la lumière solaire. En effet, l'importance de la biomasse herbacée dans les forêts galeries dégradées est surtout liée à la discontinuité de la structure de la canopée. Les forêts galeries du secteur d'étude sont soumises à d'intenses pressions pastorales caractérisées par l'émondage et l'étêtage des arbres tels que Khaya senegalensis, Afzelia africana, Pterocarpus erinaceus, par les bouviers, à l'exploitation forestière, à la carbonisation et aux activités agricoles qui imposent de façon radicale le changement physionomique de la végétation. La forte pression exercée sur cette formation a entraîné la discontinuité de la structure de la canopée, laissant de ce fait des trouées par lesquelles les rayons solaires pénètrent dans la strate herbacée. Cette forte insolation augmente la photosynthèse des espèces surtout graminéennes telles que Andropogon macrophyllus et Andropogon tectorum. Ce qui fait accroître le biovolume des espèces et augmente de ce fait leur biomasse. Par contre, le fort taux de couverture de la strate ligneuse empêche l'installation d'un important tapis herbacé car il accentue la compétition pour l'eau et les nutriments et réduit l'intensité des rayons solaires sur la strate herbacée.

Les résultats de la variation de la biomasse herbacée selon les formations végétales corroborent ceux de Mordelet et Menaut (1995) et Sawadogo et al. (2005) qui ont estimé que la diminution du taux de couverture des arbres entraîne la prolifération des thérophytes et des hémicryptophytes qui contribuent pour une large part à la biomasse herbacée. En effet, pour Semmartin et Oesterheld (1996), Lodhiyal et Lodhiyal (2003) et Sawadogo et al. (2005), la réduction de la densité des ligneux réduit la compétition pour l'eau et les nutriments et augmente l'intensité des rayons solaires sur la strate herbacée, ce qui favorise la photosynthèse.

Par ailleurs, la composition floristique a un effet significatif sur la variation de la biomasse herbacée. Les types biologiques jouent à cet effet un rôle déterminant. Ainsi, les groupements végétaux à dominance graminéenne produisent plus de biomasse herbacée que ceux dominés par les forbes. Les espèces telles que Paspalum scrobiculatum, Hyparrhenia rufa, Schizachyrium platyphyllum, Sorghastrum bipennatum et Setaria sphacelata contribuent de façon déterminante à la production de la biomasse herbacée dans les dépressions. Dans le secteur d'étude, le recouvrement moyen de ces espèces dans les dépressions est de près de $80 \%$. Cependant, sur les interfluves et les versants, ce sont plutôt Andropogon tectorum, Andropogon gayanus, Hyparrhenia involucrata, Pennisetum unisetum, Rottboellia cochinchinensis et Andropogon macrophyllus qui déterminent la productivité. Le 
recouvrement moyen de ces espèces sur les interfluves et les versants est de plus de $90 \%$.

Mais la composition floristique à elle seule ne détermine pas la variation de la biomasse herbacée. C'est plutôt le poids des espèces qui est le facteur le plus important. Ainsi, une grande richesse spécifique composée de plusieurs espèces faiblement productives fournira une biomasse moindre qu'une faible richesse spécifique composée d'espèces très productives. C'est ainsi que dans les dépressions, la biomasse produite est élevée et de variance moindre à cause du faible indice d’hétérogénéité de la végétation.

Ces résultats sont similaires à ceux de plusieurs auteurs (Lejoly et Sinsin, 1994 ; Agonyissa et Sinsin, 1998; Sinsin et al., 2000 ; Houinato et al., 2003; Wardle et Grime, 2003) qui ont estimé que l'augmentation de la productivité est surtout liée à la proportion d'espèces productives qui dépend de la composition floristique et que les groupements végétaux à dominance graminéenne produisent plus de biomasse herbacée que ceux dominés par les forbes.

Par contre le résultat concernant le rôle de la composition floristique dans la variation de la biomasse herbacée dans le secteur d'étude n'est pas conforme avec ceux de certains auteurs (Tilman et al., 1996 ; Pfisterer et Schmid, 2002 ; Kennedy et al., 2003 ; Sanderson, 2004 ; Cardinale et al., 2004 ; Szwagrzyk et Gazda, 2007). Pour ces auteurs, la grande diversité spécifique conduit à la forte productivité des écosystèmes et que la réduction de la richesse spécifique peut conduire à une faible production de biomasse.

\section{REFERENCES}

Adjanohoun EJ, Adjakidjè V, Ahyi MRA, Aké Assi L, Akoègninou A, d'Almeida J, Apovo F, Boukef K, Chadare M, Cusset G, Dramane K, Eyme J, Gassita JN, Gbaguidi N, Goudote E, Guinko S, Houngnon P, Issa LO, Keita A, Kiniffo HV, Kone-Bamba D, Musampa Nseyya A, Saadou M, Sodogandji Th, de Souza S, Tchabi A, Zinsou Dossa C, Zohoun
TH. 1989. Contribution aux Etudes Ethnobotaniques et Floristiques en République Populaire du Bénin. Agence de Coopération Culturelle et Technique: Paris; 895 p.

Adomou AC, Sinsin B, van der Maesen LJG. 2006. Phytosociological and chorological approaches to phytogeography: a mesoscale study in Benin. Syst. Geogr. Pl., 76: 155-178.

Agonyissa D, Sinsin B. 1998. Productivité et capacité de charge des pâturages naturels au Bénin. Revue Elev. Méd. Vét. Pays trop., 51(3): 239-246.

Cardinale BJ, Ives AR, Inchausti P. 2004. Effects of species diversity on the primary productivity of ecosystems: extending our spatial and temporal scales of inference. Oikos, 104: 437-450.

Djego J, Sinsin B. 2006. Impact des espèces exotiques plantées sur la diversité spécifique des phytocénoses de leur sous-bois. Syst. Geogr. Pl., 76: 191-209.

Ellis JE, Swift DM. 1988. Stability of African pastoral ecosystems, alternate paradigms and implications for development. $J$. Range Manage., 41(6): 450-459.

Faure P. 1977. Carte Pédologique de Reconnaissance de la République Populaire du Bénin à 1/200 000, Feuille de Djougou. ORSTOM: Paris; 49 p.

Garnier LKM, Dajoz I. 2001. The influence of fire on the demography of a dominant grass species of West African savannas, Hyparrhenia diplandra. J. Ecol. Oxford, 89(2): 200-208.

Gould WA, Gonzalez G, Carrero RG. 2006. Structure and composition of vegetation along an elevatioal gradient in Puerto Rico. Journal of Vegetation Science, 17: 653-664.

Hansen AJ, Rotella JJ, Kraska MPV, Brown D. 2000. Spatial patterns of primary productivity in the Greater Yellowstone Ecosystem Landscape. Ecology, 15: 505522.

Houinato M, Sinsin B, Lejoly J. 2001. Impact des feux de brousse sur la dynamique des 
communautés végétales dans la forêt de Bassila (Bénin). Acta Bot. Gallica, 148(3): 237-251.

Houinato M, Kagone H, Sinsin B. 2003. Seasonal cycle of herbaceous plant phytomass of Monts Kouffe Region in Benin. In Proceedings of the VIIth International Rangelands Congress. Durban, South Africa; 1399-1402.

Hutchinson CF. 1996. The Sahelian desertification debate: a view from the American south-west. Journal of Arid Environments, 33(4): 519-524.

IMPETUS. 2005. Benin Atlas - Research results version 0.7. IMPETUS, Remote Sensing Research Group, University of Bonn, Bonn.

Kennedy AD, Biggs H, Zambatis N. 2003. Relationship between grass species richness and ecosystem stability in Kruger National Park, South Africa African. Journal of Ecology, 41: 131140.

Keya GA. 1998. Herbaceous layer production and utilization by herbivores under different ecological conditions in an arid savanna of Kenya. Agric. Ecosyst. Environ., 69(1): 55-67.

Kokou K, Batawaila K, Akouegninou A, Akpagana K. 2000. Analyse morphostructurale et diversité floristique des îlots de forêts protégées dans la plaine côtière du sud du Togo. Etudes flor.veg. Burkina Faso, 5: 33-48.

Lejoly J, Sinsin B. 1993. China grass (Pennisetum polystachion (L.) Shult) pasture in northern Benin: Fodder species composition, productivity, feed value and grazing value. In Procceeding of the XVII International Grassland Congress ; 257-259.

Lejoly J, Sinsin B. 1994. Caractéristiques floristiques et pondérales du groupement précoce à Brachiaria lata dans les jachères soudaniennes de 8 à 32 mois du périmètre Nikki-Kalale (nord-Bénin). XIII $^{\text {th }}$ Plenary meeting AETFAT, 2: 14411452.
Lepart J. 1997. La crise environnementale et les théories de l'équilibre en écologie. In La crise environnementale 80, Larrere C, Larrere R (Eds). INRA: Paris; 131-144.

Lodhiyal N, Lodhiyal LS. 2003. Biomasse and net primary production of Bhabar Shisham Forests in Central Himalaya, India. Forest Ecology and Management, 176: $217-235$.

Marage D. 2004. Déterminisme, dynamique et modélisation spatiale de la diversité floristique dans un contexte de déprise pastorale. Application à la gestion durable des espaces montagnards sous influence méditerranéenne. Thèse de doctorat, Cemagref / Inra, France, 236 p.

Mordelet P, Menaut JC. 1995. Influence of trees on above-ground production dynamics of grasses in a humid savanna. J. Veg. Sci., 6(2): 223-228.

Orthmann B. 2005. Vegetation ecology of a woodland-savanna mosaic in central Benin (West Africa): Ecosystem analysis with a focus on the impact of selective logging. $\mathrm{PhD}$ thesis, University of Rostock, Rostock, p.148.

Osem Y, Perevolotsky A, Kigel J. 2004. Site productivity and plant size explain the response of annual species to grazing exclusion in a Mediterranean semi-arid rangeland. Journal of Ecology, 92: 297309.

Pfisterer AB, Schmid B. 2002. Diversitydependent production can decrease the stability of ecosystem functioning. Nature, 416: 84-86.

Sanderson FMA, Skinner RH, Barker DJ, Edwards GR, Tracy BF, Wedin DA. 2004. Plant species diversity and management of temperate forage and grazing land ecosystems. Crop Sci., 44: 1132-1144.

Sawadogo L, Tiveau D, Nygard R. 2005. Influence of selective tree cutting, livestock and prescribed fire on herbaceous biomass in the savannah woodlands of Burkina Faso, West Africa. 
Agriculture, Ecosystems and Environment, 105: 335-345.

Seghieri J, Floret C, Pontanier R. 1995. Plant phenology in relation to water availability- herbaceous and woody species in the savannas of northern cameroon. J. Trop. Ecol., 11: 237-254.

Semmartin M, Oesterheld M. 1996. Effect of grazing pattern on primary productivity. Oikos, 75: 431-436.

Sinsin B, Oloulotan S, Oumorou M. 1989. Les pâturages de saison sèche de la zone soudanienne du nord-est du Bénin. Rev. Elev. Méd. Vét. Pays trop., 42(2): 283288.

Sinsin B, Oumorou M, Ogoubiyi V. 2000. Les faciès à Andropogon pseudapricus des groupements post-culturaux et des savanes arbustives du nord-Bénin: dissemblance floristique et caractères communs. The Biodiversity of African Plants, 231-238.

Squires VR, Bennett F. 2004. Land capability assessment and estimation of pastoral potential of semiarid rangeland in South Australia. Arid Land Research and Management, 18: 25-37.

Szwagrzyk J, Gazda A. 2007. Above-ground standing biomass and tree species diversity in natural stands of Central Europe. Journal of Vegetation Science, 18: $555-562$.
Tilman D, Wedin D, Knops J. 1996. Productivity and sustainability influenced by biodiversity in grassland eco-systems. Nature, 379: 718-720.

Toko I, Sinsin B. 2008. Les phénomènes d'érosion et d'effondrement naturels des sols (dongas) du Parc national du $\mathrm{W}$ et leur impact sur la productivité des pâturages. Sécheresse, 19(3): 193-200.

Van der Welle MEW, Vermeulen PJ, Shaver GR, Berendse F. 2003. Factors determining plant species richness in Alaskan arctic tundra. Journal of Vegetation Science, 14: 717-720.

Wardle DA, Grime JP. 2003. Biodiversity and stability of grassland ecosystem functioning. Oikos, 100: 622-623.

Weber HE, Moravec J, Theurillat J-P. 2000. International code of phytosociological nomenclature. Journal of Vegetation Science, 11: 739-768.

White F. 1983. The vegetation of Africa. A descriptive memoir to accompany the UNESCO/AETFAT/UNSO vegetation map of Africa. UNESCO, Paris, 356 p. 